Check for updates

The BMJ

Cite this as: $B M J 2021 ; 372: n 338$ http://dx.doi.org/10.1136/bmj.n338 Published: 04 February 2021

\section{Covid-19: Israel sees new infections plummet following vaccinations}

\section{Elisabeth Mahase}

Early findings from Israel's covid-19 vaccination programme suggest that the rollout of the Pfizer BioNTech vaccine is leading to fewer new infections and is at least $50 \%$ effective 13 to 24 days after the first dose.

Israeli Ministry of Health figures, reported by the BBC, ${ }^{1}$ found that only 531 people out of almost 750 ooo fully vaccinated over 60 year olds tested positive for covid-19 (0.07\%). Of these, just 38 were hospitalised with moderate, severe, or critical disease.

The ministry analysed the records of nearly one million people between their first vaccine dose to at least seven days after the second dose. They found that there were three covid-19 deaths in vaccinated over 60 , but said it was possible they contracted the virus at an earlier stage before their immunity had time to build up.

In the period before the protection took effect, over 7000 infections were recorded, with just under 700 cases of moderate to critical illness and 307 deaths. No time scale or age group for these figures was given. The Ministry of Health said that the current data indicated that new cases and illnesses fell consistently from 14 days following the first dose.

Currently Israel has vaccinated more than $3.1 \mathrm{~m}$ people, $1.8 \mathrm{~m}$ of whom have received a second dose. Notably the country is not vaccinating certified recovered covid-19 patients, although the Ministry of Health website does not provide information on how someone is certified to have recovered. ${ }^{2}$

The website states ${ }^{3}$ that “there isn't enough information about the period of time during which a recovered coronavirus patient is considered protected, a phenomenon known as 'natural immunity.' At the first stage, individuals deemed to be recovered patients will not be vaccinated." Other exclusions include people with a history of severe allergic reactions and children under 16.

\section{Single dose efficacy}

A preprint from Israel has analysed the data of more than 500 ooo people, of whom just over 350000 had 13-24 days of follow-up post first vaccine dose, to estimate the efficacy of the Pfizer BioNTech vaccine. It found that the cumulative incidence of SARS-CoV-2 infection was $0.57 \%$ during days one to 12 and $0.27 \%$ in days 13-24. It concluded that a single dose of the vaccine was $51 \%$ effective $13-24$ days post vaccination. ${ }^{4}$

Meanwhile, researchers from the University of East Anglia reanalysed the same data and said that efficacy was "pretty much zero at day 14 , but then rose to about $90 \%$ at day 21 before levelling off." The team, who published a preprint on the findings, ${ }^{5}$ said that there was an initial surge in infection risk after having the vaccine, and suggested this could be because "people were less cautious about maintaining protective behaviours as soon as they have the injection."

They added, "What our analysis shows is that a single dose of vaccine is highly protective, although it can take up to 21 days to achieve this. The early results coming from Israel support the UK policy of extending the gap between doses by showing that a single dose can give a high level of protection."

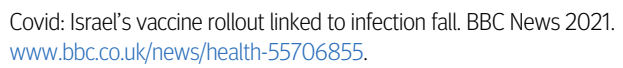

Covid: Israel's vaccine rollout linked to infection fall. BBC News 2021. www.bbc.co.uk/news/health-55706855.

2 Israel Ministry of Health. Covid-19 vaccine information. https://govextra.gov.il/ministry-of-health/covid19-vaccine/en-covid19-vaccination-information.

3 Israel Ministry of Health. Covid-19 FAQs. https://govextra.gov.il/ministryof-health/covid19-vaccine/en-covid19-vaccine-faqs.

4 Chodick G, Tene L, Patalon T, et al. The effectiveness of the first dose of BNT162b2 vaccine in reducing SARS-CoV-2 infection 13-24 days after immunization: real-world evidence.Medrxiv 2021.01.27.21250612v1 [Preprint]. 2021. www.medrxiv.org/content/10.1101/2021.01.27.21250612v1.

5 Hunter PR, Brainard JS. Estimating the effectiveness of the Pfizer covid-19 BNT162b2 vaccine after a single dose: a reanalysis of a study of "real world" vaccination outcomes from Israel.Medrxiv 2021.02.01.21250957v1 [Preprint]. 2021. www.medrxiv.org/content/10.1101/2021.02.01.21250957v1.

This article is made freely available for use in accordance with BMJ's website terms and conditions for the duration of the covid-19 pandemic or until otherwise determined by BMJ. You may use, download and print the article for any lawful, non-commercial purpose (including text and data mining) provided that all copyright notices and trade marks are retained. 